\title{
First-principles calculation of field emission from metal surfaces
}

\author{
T. Ohwaki, ${ }^{1}$ H. Ishida, ${ }^{2}$ and A. Liebsch ${ }^{1}$ \\ ${ }^{1}$ Institut für Festkörperforschung, Forschungszentrum Jülich, 52425 Jülich, Germany \\ ${ }^{2}$ College of Humanities and Sciences, Nihon University, Sakura-josui, Tokyo 156, Japan
}

(Received 13 August 2003; published 23 October 2003)

\begin{abstract}
The field-emission current from realistic metal surfaces is evaluated within the density-functional theory using the Landauer-Büttiker approach. The electronic density in the surface region and the potential barrier induced by the finite electric field are calculated self-consistently using a Green's-function embedding scheme and the full-potential linearized-augmented plane-wave method. Application of this formalism to the (100) and (111) faces of $\mathrm{Au}$ and $\mathrm{Cu}$ demonstrates the sensitivity of the field-emission current to the surface electronic structure close to the Fermi energy.
\end{abstract}

DOI: 10.1103/PhysRevB.68.155422

PACS number(s): 73.20.At, 71.15.Mb

Field emission has recently received renewed attention because of its importance for modern field-emitting devices. ${ }^{1}$ Emission from carbon nanotubes is of particular interest since they are currently being investigated for the nextgeneration flat-panel displays. ${ }^{2-5}$ The role of surface carbon atoms, adsorbed species, and of localized surface states compared to extended bulk states is so far not well understood. A microscopic description of the emitted current in terms of the electronic structure of realistic materials subject to an electric field is therefore highly desirable in order to achieve an understanding of the key parameters characterizing the emission properties and to tailor them to specific technological applications.

Earlier theoretical formulations of field emission were based on one-dimensional model potentials ${ }^{6}$ and the nonself-consistent three-dimensional layer-Kohn-KorringaRostocker approach. ${ }^{7,8}$ Transport calculations for atoms or molecules between jellium electrodes exposed to a bias potential were performed by several authors. ${ }^{9-11}$ Only recently self-consistent density functional calculations of field emission from clean and adsorbate-covered jellium surfaces were carried out. ${ }^{12}$ Also, emission from free and adsorbed finitelength carbon nanotubes ${ }^{13,14}$ and from finite slabs of graphitic ribbons ${ }^{15}$ was investigated. To our knowledge, however, field-emission calculations for realistic half-space systems are not yet available.

Here we present a formalism for the evaluation of the field-emission current from three-dimensional semi-infinite metal surfaces. The electronic structure in the vicinity of the surface exposed to an electric field is calculated selfconsistently within density-functional theory by making use of the full-potential linearized augmented plane-wave (FLAPW) method and the surface-embedded Green-function technique. ${ }^{16}$ The emission current from bulk states is evaluated using the Landauer-Büttiker formula. ${ }^{17,18}$ The surfacestate current is derived from the width of the resonance induced by the electric field. The virtue of our half space geometry is that the metal states form a continuum and that the discrete level spacing in the case of finite-range systems is avoided. This is particularly important for an accurate treatment of electron emission which is limited to a range of a few tenths of an eV below the Fermi level.
We have applied our formalism to (100) and (111) surfaces of $\mathrm{Au}$ and $\mathrm{Cu}$ in order to determine the influence of the electronic structure on the field-emission current. While for (100) surfaces the current arises solely from bulk states, in the case of (111) surfaces both bulk and surface states contribute to the total current. Despite the nearly free-electron behavior of the $\mathrm{Au}$ and $\mathrm{Cu}$ bands near the Fermi energy, the emission intensities on the (111) and (100) faces differ remarkably. They also differ significantly from the currents from equivalent jellium surfaces. Thus, the energy and momentum dependence of the electronic density, as well as its spatial distribution in the surface region, are crucial ingredients for a detailed understanding of the field-emission properties.

For the purpose of evaluating the field-emission current it is convenient to separate the surface region from the bulk and vacuum as indicated in Fig. 1. Because of the efficient screening in metals the boundary $S_{1}$ between surface and bulk regions lies typically a few atomic planes below the surface. On the vacuum side the electronic density decays rapidly even in the presence of electric fields. The surfacevacuum boundary $S_{2}$ is taken to lie at a distance above the first atomic plane where the electron density has decayed to negligible values. The main idea of the embedding theory ${ }^{16}$ is to perform a self-consistent electronic structure calculation

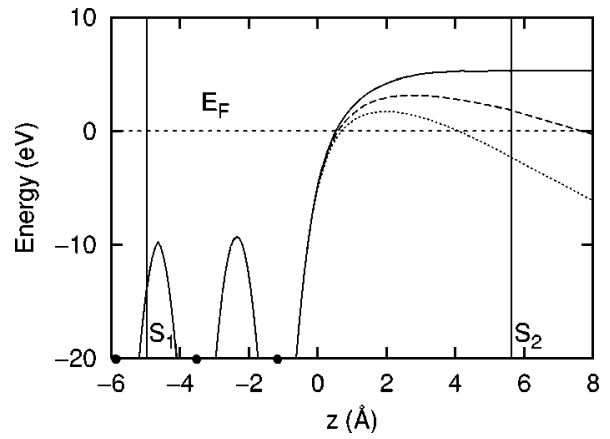

FIG. 1. Illustration of geometry used for the calculation of the conductance of the surface region in the presence of a finite electric field. $S_{1}$ and $S_{2}$ denote the bulk-surface and surface-vacuum boundaries. Solid line, laterally averaged electronic potential of $\mathrm{Au}(111)$ for $\sigma=-2 \times 10^{-3}$ a.u.; dashed line; $\sigma=-10^{-3}$ a.u.; and dotted line, $\sigma=0$. The dots denote the positions of atomic layers. 

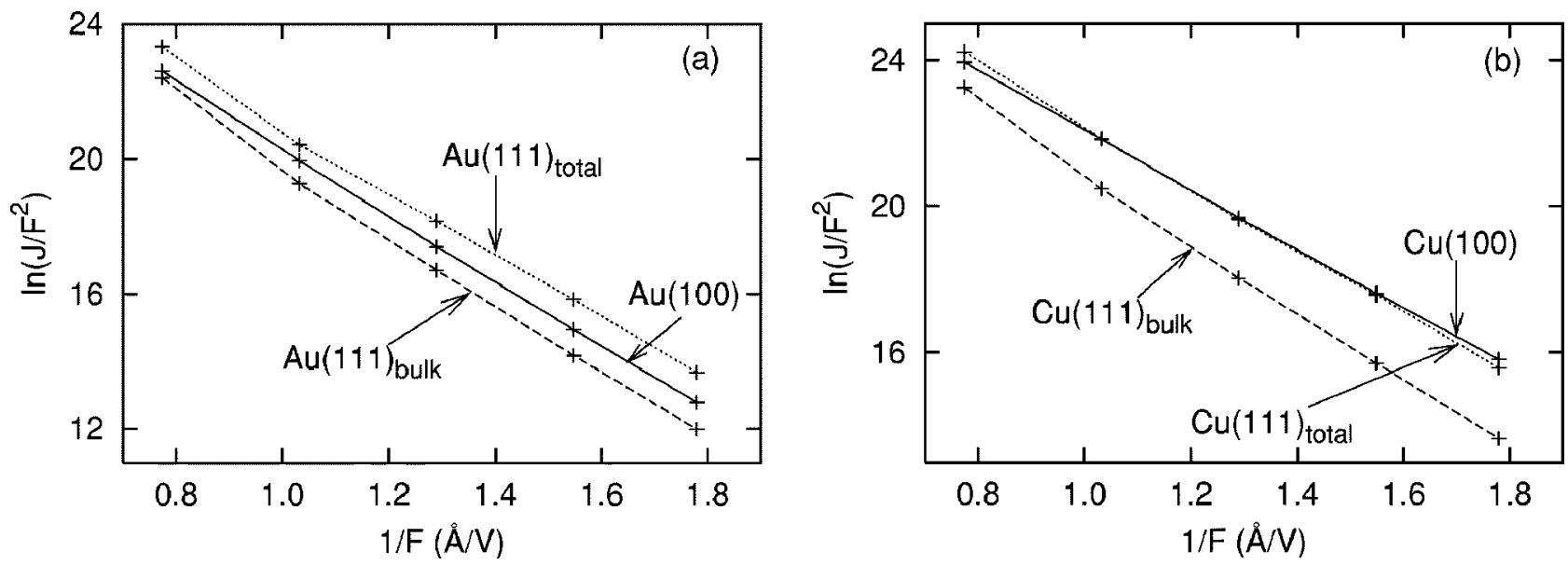

FIG. 2. Field-emission Fowler-Nordheim plots for (a) Au and (b) $\mathrm{Cu}$ surfaces. Solid curves, (100) face; dashed curves, (111) face bulk emission; and dotted curves, (111) face total emission.

in the region between $S_{1}$ and $S_{2}$. The effects of the bulk and vacuum regions are incorporated via nonlocal energydependent embedding potentials acting at $S_{1}$ and $S_{2}$. The embedding potential on the bulk side is calculated from the complex band structure of $\mathrm{Au}$ and $\mathrm{Cu},{ }^{19}$ while that on the vacuum side is expressed analytically in terms of Airy functions.

Consider first field emission from bulk states. According to the Landauer-Büttiker formula, the averaged current density may be written as

$$
\begin{gathered}
J_{b}=\int_{\mathrm{SBZ}} \frac{2}{(2 \pi)^{2}} d k_{\|} \int_{-\infty}^{E_{F}} d \epsilon g\left(\epsilon, k_{\|}\right), \\
g\left(\epsilon, k_{\|}\right)=\frac{e}{2 \pi \hbar} \sum_{i} T_{i}\left(\epsilon, k_{\|}\right),
\end{gathered}
$$

where SBZ denotes the surface Brillouin zone, $g\left(\epsilon, k_{\|}\right)$is the energy and momentum dependent conductance of the surface region, and $T_{i}\left(\epsilon, k_{\|}\right)$is the transmission probability of a oneelectron state incident from the metal interior with channel index $i$. Within the embedding scheme, $g\left(\epsilon, k_{\|}\right)$can be reformulated as ${ }^{20}$

$$
\begin{aligned}
g\left(\epsilon, k_{\|}\right)= & \frac{2 e}{\pi \hbar} \int_{S_{1}} d S_{1} d S_{1}^{\prime} \int_{S_{2}} d S_{2} d S_{2}^{\prime} G(1,2) \\
& \times \operatorname{Im} G_{S_{2}}^{-1}\left(2,2^{\prime}\right) G^{*}\left(2^{\prime}, 1^{\prime}\right) \operatorname{Im} G_{S_{1}}^{-1}\left(1^{\prime}, 1\right),
\end{aligned}
$$

where $G(1,2)$ represents the Green's function $G\left(\vec{r}_{1}, \vec{r}_{2}, \epsilon, k_{\|}\right)$ with $\vec{r}_{1}$ and $\vec{r}_{2}$ on the planes $S_{1}$ and $S_{2}$, respectively. $G_{S_{i}}^{-1}$ denotes the embedding potential at the bulk-surface or surface-vacuum boundary.

Field emission from surface states is not included in the above formalism. To evaluate the emission from the $\mathrm{Au}(111)$ and $\mathrm{Cu}(111)$ surface bands we use the following approach. In the absence of the applied field surface states have discrete energies that disperse only with $k_{\|}$. For finite fields these states couple to the continuum on the vacuum side of the barrier and acquire a finite width. Let us denote the center and the half width at half maximum of this Lorentzian profile by $E_{s}\left(k_{\|}\right)$and $\Gamma_{s}\left(k_{\|}\right)$, respectively. We now assume that the resupply of electrons into the surface state via electronelectron interactions proceeds much faster than the tunneling from this state into the vacuum. In the case of metals this assumption is well justified. ${ }^{21}$ The lifetime of the surface state at $k_{\|}$is given by $\tau_{s}\left(k_{\|}\right)=\hbar / \Gamma_{s}\left(k_{\|}\right)$. The emission current density from the surface state (electrons per sec) may then be obtained from the expression

$$
J_{s}=\int_{\mathrm{SBZ}} \frac{2}{(2 \pi)^{2}} d k_{\|} \frac{e}{\hbar} \Gamma_{s}\left(k_{\|}\right) \theta\left[E_{F}-E_{s}\left(k_{\|}\right)\right] .
$$

The $\mathrm{Au}(111)$ and $\mathrm{Cu}(111)$ surface bands in the energy gap at $L$ have nearly quadratic dispersion with $k_{\|}$. In the absence of the field their minima at $k_{\|}=0$ are located at $-0.38 \mathrm{eV}$ and $-0.54 \mathrm{eV}$ below $E_{F}$, respectively. With increasing electric field the surface band shifts slightly towards larger binding energies.

The numerical calculations are carried out by extending a surface-embedded Green's-function FLAPW code developed by one of the authors ${ }^{22}$ to charged metal surfaces. In the present work we consider unreconstructed surfaces without lattice relaxations. Two atomic layers are included in the embedded region. Figure 1 shows the self-consistent potential of $\mathrm{Au}(111)$ for three values of $\sigma$, the surface charge per unit area. As a result of the efficient screening of the applied field these potentials differ only outside the outermost Au layer.

Figure 2 shows the field-emission current for (100) and (111) surfaces of $\mathrm{Au}$ and $\mathrm{Cu}$ as a function of the strength of the electric-field $F$. To illustrate the validity of the Fowler-

TABLE I. Field-emission current densities $J\left(10^{8} \mathrm{~A} / \mathrm{m}^{2}\right)$ from (111) and (100) faces of $\mathrm{Au}$ and $\mathrm{Cu}$, and from equivalent jellium surfaces; $\sigma=-10^{-3}$ a.u.

\begin{tabular}{ccccccc}
\hline \hline & $(111)_{\text {bulk }}$ & $(111)_{\mathrm{ss}}$ & $(111)_{\text {total }}$ & $(100)_{\text {total }}$ & $(111)_{\text {jell }}$ & $(100)_{\text {jell }}$ \\
\hline$J_{\mathrm{Au}}$ & 0.60 & 2.54 & 3.14 & 1.29 & 2.49 & 2.26 \\
$J_{\mathrm{Cu}}$ & 2.77 & 15.1 & 17.9 & 18.8 & 25.4 & 49.3 \\
\hline \hline
\end{tabular}




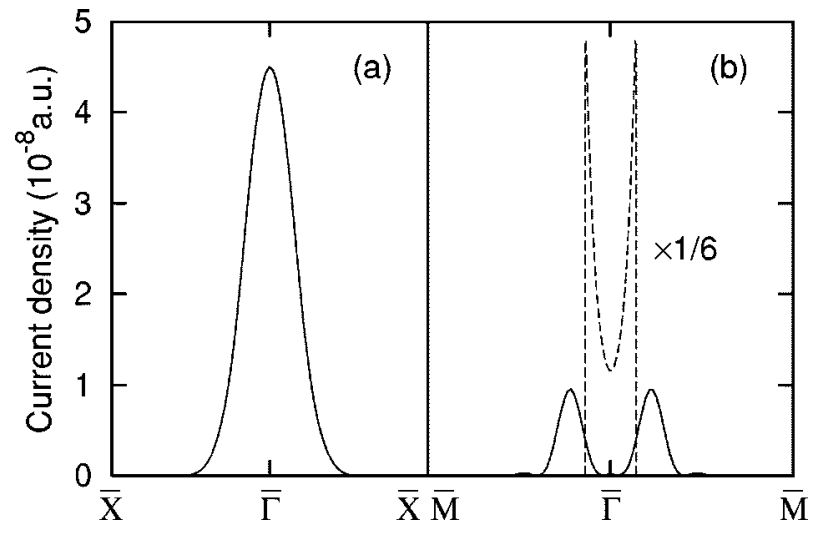

FIG. 3. Field-emission current density for (a) $\mathrm{Au}(100)$ and (b) $\mathrm{Au}(111)$ as a function of $k_{\|}$for $\sigma=-10^{-3}$ a.u. The (100) current has a cusp at the center of the surface Brillouin zone, as expected for free-electron systems. In contrast, the (111) bulk current is maximal at finite $k_{\|}$because of the band gap at the $L$ point. The (111) surface current is maximal at $k_{\|}$where the surface state crosses the Fermi level.

Nordheim relation we plot $\ln \left(J / F^{2}\right)$ versus $1 / F$. The results demonstrate that this linearity is indeed satisfied for bulk and surface currents up to large field strengths corresponding to induced surface charge densities of about $\sigma=-2$ $\times 10^{-3}$ a.u. $(1 / F=0.77 \AA / \mathrm{V})$.

Table I provides the absolute currents for $1 / F$ $=1.55 \AA / \mathrm{V}$. For comparison we also give the results for equivalent jellium surfaces where an average ionic pseudopotential in the metal interior is introduced to reproduce the calculated $\mathrm{Au}$ and $\mathrm{Cu}$ work functions. Several aspects of these data are noteworthy.

(i) Although the $\mathrm{Au}(100)$ and (111) surfaces have nearly identical work functions $\left(\Phi_{\mathrm{Au}}(100)=5.67 \mathrm{eV}, \Phi_{\mathrm{Au}}(111)\right.$ $=5.71 \mathrm{eV})$, the $(100)$ emission is more than twice as strong as the (111) bulk current. However, the Au(111) surface current is about four times larger than the bulk contribution so that the total $\mathrm{Au}(111)$ current is much larger than the $\mathrm{Au}(100)$ emission.

(ii) The picture for $\mathrm{Cu}$ is quite different since now there is a larger difference between the (100) and (111) work functions $\left(\Phi_{\mathrm{Cu}}(100)=5.01 \mathrm{eV}, \quad \Phi_{\mathrm{Cu}}(111)=5.19 \mathrm{eV}\right)$. Thus, even though, like in $\mathrm{Au}(111)$, the $\mathrm{Cu}(111)$ surface emission is much larger than the bulk emission, the total (111) current remains smaller than the (100) emission.

(iii) The important role played by the work function becomes also evident by comparing the $\mathrm{Au}$ and $\mathrm{Cu}$ emission currents. While the bulk and surface contributions for $\mathrm{Cu}(111)$ are about six times larger than for $\mathrm{Au}(111)$ (the work functions differ by $0.52 \mathrm{eV}$ ), the $\mathrm{Cu}(100)$ emission is almost 15 times larger than the $\mathrm{Au}(100)$ current (the workfunction difference is $0.66 \mathrm{eV}$ ). The large variation among these results underlines the importance of the microscopic details of the surface electronic properties for the fieldemission characteristics. ${ }^{23}$

(iv) Although close to the Fermi level both $\mathrm{Au}$ and $\mathrm{Cu}$ are nearly free-electron systems the (111) and (100) fieldemission currents differ significantly from those of the equivalent jellium surfaces, despite identical work functions.

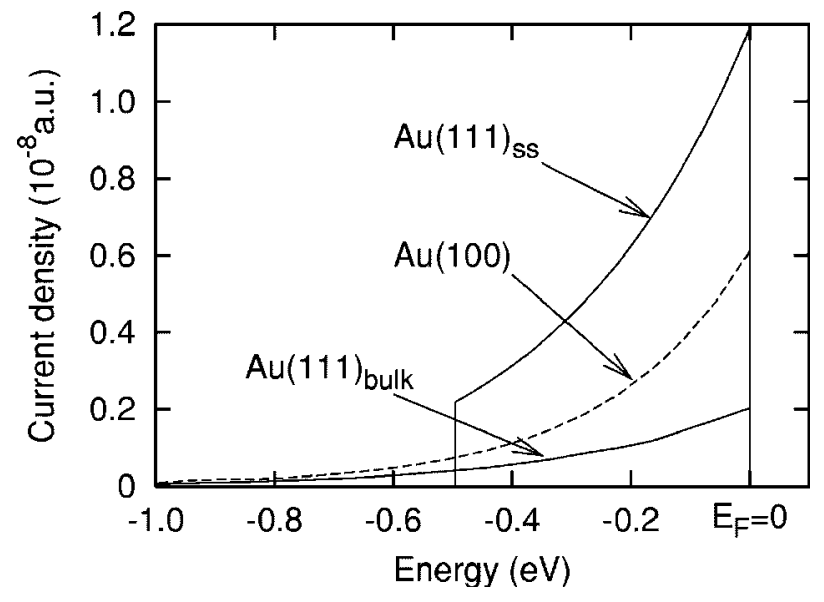

FIG. 4. Energy dependence of field-emission current density for (100) and (111) faces of Au for $\sigma=-10^{-3}$ a.u. Solid curves, (111) bulk and surface currents and dashed curve, (100) current. The lower cutoff of the surface-state contribution corresponds to the minimum of $E_{s}\left(k_{\|}\right)$at $\bar{\Gamma}$.

For instance, the $\mathrm{Au}$ and $\mathrm{Cu}(100)$ jellium currents overestimate the actual $\mathrm{Au}(100)$ and $\mathrm{Cu}(100)$ currents by a factor of $2-3$. Evidently, the $s d$ hybridization near $E_{F}$, and the fact that the $s p$ electrons must avoid the large core regions occupied by the $d$ states, strongly reduces the probability for transmission across the surface barrier. The (111) bulk and total currents of $\mathrm{Au}$ and $\mathrm{Cu}$ also differ appreciably from the jellium values.

To illustrate the difference between bulk and surfaceemission properties we show in Fig. 3 the parallel momentum variation of the Au current density. As expected for the free-electron behavior on the (100) face, its emission density is largest at the center of the surface Brillouin zone because of the presence of the $s p$ band at $E_{F}$ near $k_{\|}=0$. For $\mathrm{Au}(111)$, on the other hand, there are no states near $E_{F}$ at

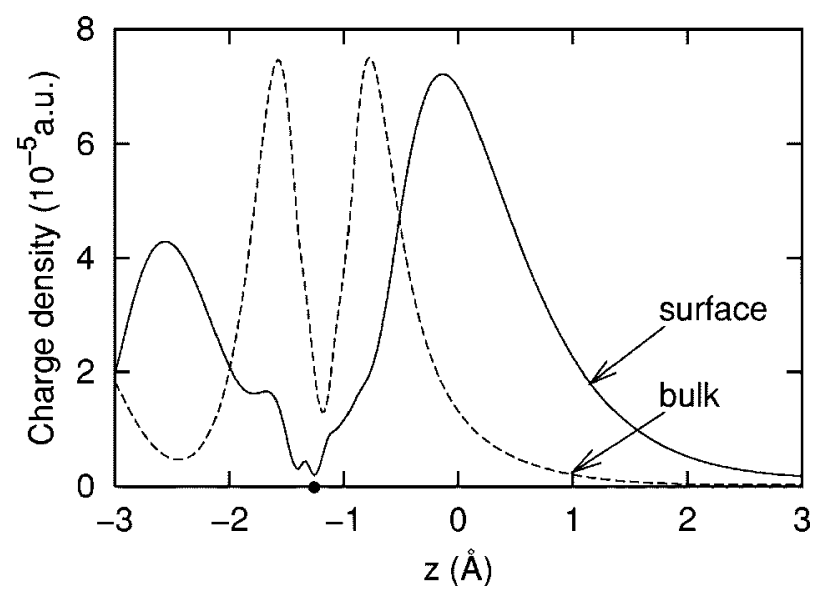

FIG. 5. Planar averaged spatial distribution of valence charge density of $\mathrm{Au}(111)$ bulk and surface states at $\bar{\Gamma}(\sigma=-2$ $\times 10^{-2}$ a.u.). The charge density of the bulk states corresponds to the energy region from -2.46 to $-1.11 \mathrm{eV}$ below $E_{F}$; the density of the surface state to the window from -0.84 to $-0.43 \mathrm{eV}$. The dot denotes the position of the first-layer atom. 
small $k_{\|}$because of the band gap at $L$. Accordingly, the current density from bulk states nearly vanishes at $\bar{\Gamma}$ and reaches its maximum at finite $k_{\|}$. Since the overall emission intensity decays exponentially with increasing $k_{\|}$, the $\mathrm{Au}(111)$ bulk current is much smaller than that for $\mathrm{Au}(100)$. According to Fig. 3(b) the $\mathrm{Au}(111)$ surface current is much larger than the bulk current. The origin of this effect will be discussed below. Here we note that the (111) emission reaches its maximum not at $\bar{\Gamma}$ but where the surface band crosses the Fermi energy. Thus, the current diminishes more rapidly with increasing binding energy than with increasing $k_{\|}$.

Figure 4 compares the energy variation of the Au (111) and (100) emission currents. The bulk contribution is obtained from the momentum integrated conductance $g\left(\epsilon, k_{\|}\right)$, while the surface contribution follows from Eq. (4) by replacing $\theta\left[E_{F}-E_{s}\left(k_{\|}\right)\right]$by $\delta\left[\epsilon-E_{s}\left(k_{\|}\right)\right]$. All contributions diminish exponentially with increasing binding energy, so that the total current essentially originates within a few tenths of an $\mathrm{eV}$ below $E_{F}$. The (111) bulk contribution is much smaller than for the (100) face because of the absence of propagating bulk states near $\bar{\Gamma}$ [see Fig. 3(b)]. The largest contribution stems from the (111) surface current. Qualitatively similar results are found for $\mathrm{Cu}$.

To illustrate the origin of the remarkably large $\mathrm{Au}(111)$ surface-state current we show in Fig. 5 the planar averaged spatial distribution of valence charge density for $\mathrm{Au}(111)$ bulk and surface states for $\bar{\Gamma}$. The important point here is that the surface-state density extends much farther into the vacuum than the bulk density. Thus, the effective width of the tunneling barrier is smaller than that for propagating bulk states. Clearly, therefore, the field-emission characteristics do not depend only on the energy and parallel momentum variation of electronic states. Their spatial location with respect to the surface barrier is an additional decisive factor.

In summary, we have presented a formalism for the evaluation of field-emission currents from realistic metal surfaces. The electronic density in the presence of the electric field is calculated self-consistently using the FLAPW embedding scheme and density-functional theory. Emission from bulk states is derived using the Landauer-Büttiker approach, whereas surface-state currents are obtained from the resonance width induced by the electric field. Application of this formalism to surfaces of $\mathrm{Au}$ and $\mathrm{Cu}$ revealed a remarkable sensitivity of the field-emission current to the surface electronic structure. Although both metals exhibit nearly freeelectron behavior near $E_{F}$, the bulk currents for the (111) and (100) faces differ strongly, and the (111) surfaces exhibit very large surface-state currents. Moreover, the results differ appreciably from the equivalent jellium surfaces. The emission characteristics therefore intimately reflect the microscopic electronic properties near the surface. Similar conclusions should apply to other materials. We expect our formalism to be useful also for surfaces of carbon systems.
${ }^{1}$ A.A. Talin, K.A. Dean, and J.E. Jaske, Solid-State Electron. 45, 963 (2001).

${ }^{2}$ W.A. de Heer, A. Chatelein, and D. Ugarte, Science 270, 1179 (1995).

${ }^{3}$ Y. Saito et al., Nature (London) 389, 554 (1997).

${ }^{4}$ W.B. Choi et al., Appl. Phys. Lett. 75, 3129 (1999).

${ }^{5}$ A.G. Rinzler et al., Science 269, 1550 (1995).

${ }^{6}$ For a review, see J.W. Gadzuk and E.W. Plummer, Rev. Mod. Phys. 45, 487 (1973).

${ }^{7}$ A. Modinos and N. Nicolaou, Phys. Rev. B 13, 1536 (1976); A. Modinos, J. Phys. C 9, 3867 (1976); A. Modinos, Field, Thermionic, and Secondary Electron Emission Spectroscopy (Plenum, New York, 1984).

${ }^{8}$ P. Soven, E.W. Plummer, and N. Kar, CRC Crit. Rev. Solid State Sci. 1, 111 (1976).

${ }^{9}$ J. Neugebauer and M. Scheffler, Surf. Sci. 287/288, 572 (1993).

${ }^{10}$ N.D. Lang, Phys. Rev. B 52, 5335 (1995).

${ }^{11}$ K. Hirose and M. Tsukada, Phys. Rev. B 51, 5278 (1995).
${ }^{12}$ T. Gohda and S. Watanabe, Phys. Rev. Lett. 87, 177601 (2001).

${ }^{13}$ S. Han and J. Ihm, Phys. Rev. B 61, 9986 (2000).

${ }^{14}$ Ch. Adessi and M. Devel, Phys. Rev. B 62, R13 314 (2000).

${ }^{15}$ K. Tada and S. Watanabe, Phys. Rev. Lett. 88, 127601 (2002).

${ }^{16}$ J. Inglesfield, J. Phys. C 14, 3795 (1981).

${ }^{17}$ R. Landauer, Philos. Mag. 21, 863 (1970).

${ }^{18}$ M. Büttiker, Phys. Rev. Lett. 57, 1761 (1986).

${ }^{19}$ D. Wortmann, H. Ishida, and S. Bluegel, Phys. Rev. B 65, 165103 (2002).

${ }^{20}$ D. Wortmann, H. Ishida, and S. Bluegel, Phys. Rev. B 66, 075113 (2002).

${ }^{21}$ J.W. Gadzuk, J. Vac. Sci. Technol. 9, 591 (1971).

${ }^{22}$ H. Ishida, Phys. Rev. B 63, 165409 (2001).

${ }^{23}$ Note that the calculated work functions are between $0.2 \mathrm{eV}$ and $0.4 \mathrm{eV}$ larger than the measured ones. Nevertheless, the trend between $\mathrm{Au}$ and $\mathrm{Cu}$, and between their (100) and (111) faces, is the same as in the experiment. 